\title{
Visual disturbances - a borderline between ophthalmology and neurology
}

\author{
Alexandra Doina Boangiu', Gabriela Mihailescu ${ }^{1,2}$ \\ ${ }^{1}$ Neurology Department, Colentina Clinical Hospital, Bucharest, Romania \\ ${ }^{2}$ Neurology Department, "Carol Davila" University of Medicine and Pharmacy, Bucharest, Romania
}

\begin{abstract}
Visual acuity loss can be due to eyeball diseases or to lesions of the optic nerves, visual pathway or cortical projection of vision. The differential diagnosis can be challenging and though patients go fi rst to the ophthalmologist, there is not always the ophthalmologist who can diagnose and treat the patient. For a great number of causes/diseases, a neurologist should also see the patient, even if there is a transient or persistent monocular or binocular visual acuity loss a disturbance of the visual fi eld or of the perception of colours. Visual acuity loss can be more than this. It can be a symptom or a sign of a neurological or systemic disease and a rapid diagnosis and specifi c treatment are mandatory in order to treat the symptoms and improve the patient's quality of life.
\end{abstract}

Keywords: visual acuity loss, retinopathies, optic neuritis, blindness, visual field disturbances

\section{INTRODUCTION}

The visual system assures the most important integration of the body in the surrounding world, and this is proven by its cortical projections and connections with the majority of the areas into the central nervous system. A visual control is needed for movement, coordination, praxia, reading, writing, playing an instrument, painting (recognising colours, the form of the objects), driving a car, spatial orientation, executive functions and even for the behaviour.

The visual system is represented by the eyeball, the optic nerve and the visual pathway with its connections and the cortical projection. The extrinsic muscles of the eyeball innervated by the cranial nerves (nerves III, IV and VI - comune oculomotor, trochlear and abducens) assure a clear binocular vision. The pathological lesions affecting the eyeball (and there are not few) are diagnosed and treated by the ophthalmologist. Lesions affecting any other part of this pathway can be subject of a team-work or a problem to be solved by the neurologist, as well as diplopia while using both eyes (monocular diplopia beeing the subject of an ophtalmologic examination).
Functional visual loss (a decrease in visual acuity and/or visual field) is usually sending the patient first to the ophthalmologist but, if lenses do not correct the low vision, if the intraocular pressure is not high enough to explain concentric loss of the visual field or if no other ocular organic causes can explain the pathological changes, then, the patient has to be referred to a neurologist, as the cause is not an ophthalmological one, but can be part of a neurological or a systemic disease (1).

Visual acuity loss is a frequent complaint. Children and teenagers can be diagnosed with traumatic or non-traumatic conditions, as congenital disturbances, amblyopia, myopia or hypermetropia, retinitis pigmentosa, prematurity retinopathy, hypoplasia of the optic nerve, tumours of the optic nerve or suprasellar tumours. In adults, ophthalmologists are used to diagnose cataract, glaucoma, diabetic retinopathy or macular degenerescence, but vascular causes (as the occlusion of the central retinal artery or vein) or inflammatory and demyelinating lesions of the optic nerve are not rare at all (1).

The visual acuity changes can be caused by refractive errors, opacification of the transparent media of the eye (cornea, aqueous humour, crystalline 
lens, vitreous humour), by lesions of the retina (detachment, inflammation, infection, vascular occlusion, vasculitis, tumours) or by lesions of the optic nerve or optic pathways and can be monocular or binocular, with an acute or gradual onset, episodic or permanent.

\section{OVERVIEW OF DISEASES}

Transient monocular blindness has most frequent a vascular cause, and is known as amaurosis fugax. There is an acute loss of vision that can be partial or complete, lasting for some seconds to minutes. The most frequent causes are the stenosis of the internal carotid artery with secondary retinal/ choroidian hypoperfusion, giant cells arteritis, dissection of the internal carotid artery. In this case, the patient has to be referred as soon as possible to a neurologist for further investigation, as this can be a sign of a severe vascular systemic disease, announcing a future stroke (2).

Another cause of episodes of monocular transient blindness, with duration of seconds, precipitated by postural changes or Valsalva manoeuvres is the optic disc edema (papilledema). The papilledema can be bilaterally, asymmetric, secondary to intracranial hypertension, even if the visual loss is transient monocular, and excepting these episodes, the visual acuity is normal. The optic disc is elevated, hyperaemic, imprecisely delimitated and the veins are turgescent. The visual field can be normal, the blind spot can be larger or it can be concentric reduced. The patient should be investigated by cerebral Magnetic Resonance Imaging (MRI) in order to diagnose tumours, hydrocephalus, cerebral sinus vein thrombosis. If this imaging test shows no pathological findings, an infectious pathology has to be excluded by examination of the cerebrospinal fluid (meningitis, encephalitis) (2).

Other causes of transient monocular blindness are: repeated angle closure glaucoma attacks, dis- cal abnormalities (drusen), coloboma iridis, orbital tumours. In the demyelinating optic neuropathies, the Uhthoff phenomenon is described, with transient visual loss during physical exercises or exposure to excessive heat (3).

Cause of transient binocular blindness are described in Table 1.

Monocular persistent visual acuity loss can have an ocular cause or can be related to the optic nerves, extending to the junction with the optic chiasm (optic neuropathies) (2).

The most frequent causes of ocular disturbances are presented in Table 2.

The borderline between ophthalmology and neurology is represented by the differential diagnosis between macular and optic nerve disturbances (Table 3).

The most frequent retina diseases are:

1. Serous retinopathy - leakage of serous fluid in the sub-retinal space, causing detachments of the retinal layers. It mostly affects males in the decades 5 and 6 of age, is considered idiopathic, but can be exacerbated by stress or use of corticosteroids. The onset is quite acute, not painful, with central vision loss and metamorphopsia. The optic disc is normal and ocular computed tomography (OCT) is showing retinal changes. The symptoms may disappear within 1 to 6 months without treatment, but sometimes intraocular injections with Avastin or LASER treatment are required to seal the detached retinal areas (1);

2. Age related macular degeneration (AMD) affects both eyes and is progressive. The drusen (white-yellowish deposits with unclear limits) is an early sign and it is associated with hypo- or hyperpigmentation of the retinal pigment epithelium. The central vision is initially distorted and then visual acuity is lost progressively. Two types are described: the wet and the dry type (1);

3. Retinal detachments - central visual acuity loss and afferent pupillary defect if the central part of the retina is affected. Photopsia can frequently

TABLE 1. Causes of binocular transient blindness (2)

\begin{tabular}{|l|l|}
\hline Migraine aura & $\begin{array}{l}\text { Scin llant scotoma which can progress to complete congruous hemianopia } \\
\text { Followed or not by pulsa le (throbbing) headache }\end{array}$ \\
\cline { 1 - 2 } $\begin{array}{l}\text { Transient ischemic a ack in the basilar or } \\
\text { posterior cerebral arteries }\end{array}$ & $\begin{array}{l}\text { Older pa ents } \\
\text { Acute onset } \\
\text { If present, headache is concomitantly with the visual field disturbance }\end{array}$ \\
\cline { 1 - 2 } Edema of the op c disc & Causes less frequent \\
\cline { 1 - 2 } Occipital focal a acks & $\begin{array}{l}\text { Head Trauma } \\
\text { Posterior reversible encephalopathy (las ng encephalopathy } \\
\text { hours-days) }\end{array}$ \\
\hline
\end{tabular}


TABLE 2. Most frequent disturbances of the transparent media of the eye (1)

\begin{tabular}{|c|c|c|}
\hline \multirow[t]{2}{*}{ Cornea } & corneal scars & $\begin{array}{l}\text { eye trauma } \\
\text { post-infec on with Herpes simplex, Chlamydia trachoma s }\end{array}$ \\
\hline & corneal deposits & $\begin{array}{l}\text { calcium (hyperparathyroidism, vitamin D intoxica on, sarcoidosis) } \\
\text { copper (Wilson Disease) } \\
\text { arcus senilis (dyslipidemia - associated with xantelasma) } \\
\text { crystallines (mul ple myeloma, cryoglobulinemia) }\end{array}$ \\
\hline Anterior chamber & ocular hyphema & presence of blood in the anterior chamber \\
\hline \multirow[t]{2}{*}{ Crystalline } & cataract & $\begin{array}{l}\text { senile } \\
\text { diabetes mellitus - deposits of sorbitol in the crystalline } \\
\text { galactosemia - glycerol } \\
\text { hypoparathyroidism } \\
\text { long term cor cotherapy } \\
\text { radiotherapy } \\
\text { Steinert myotonic dystrophy } \\
\text { Wilson Disease (rare cases) }\end{array}$ \\
\hline & subluxa on & $\begin{array}{l}\text { syphilis } \\
\text { Marfan syndrome } \\
\text { homocys nuria }\end{array}$ \\
\hline \multirow[t]{2}{*}{ Vitreous body } & haemorrhage & $\begin{array}{l}\text { prolifera ve re nopathy } \\
\text { re nal vein occlusion } \\
\text { Terson syndrome-following subarachnoid haemorrhage } \\
\text { head trauma } \\
\text { senile macular degenera on }\end{array}$ \\
\hline & opacifica on & $\begin{array}{l}\text { condensa on of vitreous collagen fibrils } \\
\text { lymphomatous infiltra on }\end{array}$ \\
\hline
\end{tabular}

TABLE 3. Differential diagnosis between macular disturbances and optic neuropathy (2)

\begin{tabular}{|l|l|l|}
\cline { 2 - 3 } \multicolumn{1}{c|}{} & Macular disturbances & Optic nerve disturbances \\
\hline Central vision loss & Yes & Yes \\
\hline Central scotoma & Yes & Yes \\
\hline Colour vision disturbances & Yes - blue-yellow & Yes - red-green \\
\hline Pain & rare & Yes-increased by eye movements in some op cneuropathies \\
\hline Distorted vision & Yes & No \\
\hline Afferent pupillary defect & No & Yes \\
\hline
\end{tabular}

precede the detachments. The risk factors are severe myopia, recent intraocular surgery, eye trauma, family history of retinal detachments (2);

4. Central retinal artery occlusion (CRAO) suddenly, not painful, onset of unilateral blindness (if the cause is a dissection of the internal carotid artery or an arteritis with giant cells). The retina is white, opaque, edematiated and sometimes the thrombus can be seen (,cherry red spot macula"). Sometimes, the local intra-arterial thrombolysis can have good therapeutic results (4);

5. Central retinal vein occlusion (CRVO) - subacute monocular variable loss of visual acuity that sometimes can spontaneously recover. Retinal veins are dilated, tortuous and cotton-wool spots, macular edema and optic disc edema may be associated to extensive haemorrhages in the posterior pole giving the "blood and thunder appearance" (4).

The retinal vessel occlusions are affecting the layer of ganglionic cells and this can generate afferent pupillary defect;
6. Diabetic Retinopathy - is a frequent cause of blindness; it is also common in patients with stroke. The retinal ischemia is diffuse, including the macula, which leads to central vision loss. Secondary to ischemia, there is a process of neovascularisation that will eventually be responsible for haemorrhages and retinal detachments. The treatment can be done using laser photocoagulation or intravitreal injections with anti-VEGF agents, but also by maintaining glycaemic control and reducing the cardiovascular risk factors (5);

7. Hypertensive retinopathy - is usually the result of chronic arterial hypertension; it is usually asymptomatic, but the retinal damage may produce vision loss. The retinal vascular changes are usually bilaterall, but asymmetric, and include arterial narrowing, irregularity of the vessels, microaneurysms, cotton wool spots, arteriovenous nicking. However, in patients with acute hypertensive retinopathy, there are retinal haemorrhages, macular edema, retinal vessels spasms, cotton wool spots, optic nerve edema. Sometimes, there is isolated op- 
tic nerve edema, thus it's important to assess arterial blood pressure in patients with headache and bilateral optic nerve edema. There can be choroidal ischemia, ischemic optic neuropathy which can lead to permanent vision loss. In such cases, intravitreal injection with bevacizumab is helpful, improving the visual outcome (6).

Unilateral optic nerve neuropathies - can be generated by inflammation, ischemia or compression:

\section{A. Inflammatory optic neuropathies}

1. Demyelinating - are more frequent in young adults, especially women. The demyelinating optic retrobulbar neuritis (NORB) is rapidly progressive, unilaterally, (sometimes bilaterally, during the same episode or most frequent at some intervals of time) and can be inconstantly associated with orbital pain induced by eye movement or eye compression. The Uhthoff phenomenon representing the increase of visual loss due to physical exercise or exposure to heat is characteristic. The afferent pupillary defect is present. The fundoscopic eye exam shows a normal optic disc and only in the case of an associated papilitis the disc shows edema (7). The vision prognostic is good, spontaneous remission can be seen in some weeks to months. Intravenous pulse-therapy with Solumedrol may help recovery. NORB can be the first clinical sign of multiple sclerosis, that's why these patients are obliged to be seen by a neurologist and complete the investigations with a cerebral and spinal cord MRI, in order to look for other demyelinating lesions of the central nervous system and with a cerebrospinal fluid examination to look for oligoclonal bands and calculate the index immunoglobulins/albumin. Despite the visual loss recovery, in most of the patients, some degree of optic atrophy rests and the optic disc is pale in its temporal region. The Visual evoked potentials are a sensitive tool to diagnose a NORB, even if it is asymptomatic (7). In the cases when the optic neuritis is recurrent, bilaterally and is associated with transverse myelitis and the visual loss is not recovered during a month, the diagnosis of optic neuromyelitis (Devic) must be taken into account, having a poorer prognostic than the optic neuritis in multiple sclerosis. Optic neuromyelitis can also be seen in systemic infectious diseases (viral hepatitis, tuberculosis, syphilis, borreliosis) and in autoimmune diseases (systemic erythematous lupus, myasthenia gravis, autoimmune thyroiditis). Antibodies anti-aquaporin 4 can be found in the serum of up to $80 \%$ of the patients with optic neuromyelitis (8).
2. Autoimmune - recurrent episodes of optic neuritis responsive to corticosteroid therapy, most frequently in women. During the attack, the optic nerve disc is slightly edematiated. If this disease is not properly diagnosed from the onset of symptoms it can evolve to optic nerve atrophy and severe visual loss (9).

\section{Infectious}

a. Neuroretinitis - is affecting young adults who can recall a viral prodrome. The visual loss can be variable and may associate afferent pupillary defect. The patients complain of central or centrocecal scotoma and at fundoscopy they present optic disc edema (that can last 3 months) and stellate maculopathy (that can last 1 year), sometimes associating peripapillary retinal detachments (10). The recovery of visual loss normally starts some weeks after the onset of the disease, and can be incomplete if there are recurrent episodes leaving permanent sequellae (11). A frequent cause of neuroretinitis is the infection with Bartonella henselae (12);

b. Syphilis - caused by Treponema pallidum, is a disease with sexual transmission or can be transmitted from mother to her foetus. The optic nerve is affected in the late phases (secondary or tertiary) of syphilis, called neurosyphilis. The patients complain of central visual loss and dyschromatopsia. The optic disc at fundoscopy can have a normal appearance, can show atrophy or oedema and may associate stellate maculopathy, characteristic for neuroretinitis. Cerebral MRI may be normal, but cerebrospinal fluid analysis using treponemal and non-treponemal tests can establish the diagnosis. Penicillin is the specific treatment and sometimes corticosteroids may be associated (13);

c. Borrelia burgdorferi - the optic neuropathy is usually characterised by painless visual loss, bilateral papillitis and central scotoma (14). Improvement is possible with specific antibiotherapy (15);

d. Mycobacterium tuberculosis - the optic involvement may be due to tuberculomas compressing the optic chiasm or causing optico-chiasmatic arachnoiditis with secondary optic nerve inflammation. There may be pain, the vision loss may be gradual or rapid and the optic disc is normal, edematous, or atrophic. The diagnosis is confirmed by bacteria detection. A new diagnostic technique is the nested PCR assay. The treatment includes 
TABLE 4. Optic nerve neuropathies associated to systemic diseases

\begin{tabular}{|l|l|}
\hline Sarcoidosis & $\begin{array}{l}\text { systemic granulomatous inflamma on } \\
\text { recurrent visual disturbances when reducing/stopping cor cotherapy } \\
\text { females are more affected } \\
\text { op c nerve disc is normal, atrophic or oedematous } \\
\text { central scotoma, al tudinal defect of the visual field or homonymous hemianopia } \\
\text { associated pulmonary disturbances } \\
\text { cerebral MRI - op c nerves and meninges with increased intensity } \\
\text { in case of suspicion - lacrimal gland biopsy } \\
\text { Treatment - cor cotherapy }\end{array}$ \\
\hline Wegener granulomatosis & $\begin{array}{l}\text { pa ents 40-50 years of age, male. BUT op c neuri s is more frequent in women. } \\
\text { orbital signs are associated - proptosis, pain, disturbances in oculomotricity } \\
\text { lung and kidney disturbances } \\
\text { c-ANCA present in the serum } \\
\text { Treatment - cor cotherapy in big doses, cyclophosphamide, rituximab }\end{array}$ \\
\hline Sjögren syndrome & $\begin{array}{l}\text { keratoconjunc vi s, xerostomia, Sicca syndrome } \\
\text { associates op c neuromieli s }\end{array}$ \\
\hline Systemic erythematous lupus & $\begin{array}{l}\text { many other organs are affected } \\
\text { not painful, subacute, severe visual loss } \\
\text { An bodies an ds-DNA, an -Smith, an phospholipidic } \\
\text { cor cotherapy } \\
\text { cyclophosphamide, cyclosporine, methotrexate, azathioprine }\end{array}$ \\
\hline Paraneoplas c & \begin{tabular}{l} 
associated with small cell lung cancer, B cell lymphoma, prostate cancer, renal carcinoma \\
\hline
\end{tabular}
\end{tabular}

ethambutol, rifampicin, streptomycin, isonicotinic acid hydrazide and pyrazinamide (16). It is important to mention that anti-tuberculous agents, like ethambutol, may cause vision loss (17).

\section{Optic nerve neuropathies associated to sys- temic diseases.}

B. Acute ischemic optic neuropathy - diagnosed in patients over 50 years of age. It has an acute onset, without pain and progresses in some hours or days. Two types are described: anterior ( $90 \%$ of cases), with oedematous optic nerve disc, and posterior (arteritic), with normal optic nerve disc. There is also a classification of the ischemic optic neuropathies taking into account the presence of arteritis with giant cells. The risk factors are blood pressure drop during heart surgery, systemic haemorrhage, renal dialysis, severe anaemia, hypercoagulability, radiotherapy. The treatment of the non-arteritic form is based on the control of the risk factors and exclusion of the presence of arteritis with giant cells. The arteritic form, present in older patients, associates masticatory claudication, myalgia, weight loss, headache. Laboratory tests will show an inflammatory syndrome with high values of the protein $\mathrm{C}$ and the biopsy of the temporal artery that will establish the diagnosis. Corticotherapy consists of metilprednisolone iv $1 \mathrm{~g}$ /day 3-5 days followed by prednisone $1 \mathrm{mg} / \mathrm{kg} /$ day for with progressive lowering of the dose is helpful (4).

C. Compressive/infiltrative optic neuropathy meningioma in adults, glioma in children. The onset is progressive, not painful, colour vision is affected and can be associated with other malignancies or neurofibromatosis (2).

Persistent binocular visual acuity loss is caused by lesions of the retina in both eyes, of both optic nerves, optic chiasma, retrochiasmatic visual pathways (Table 5).

TABLE 5. Causes of persistent binocular visual acuity loss (2), (18)

\begin{tabular}{|c|c|c|}
\hline Re nopathies & Bilaterally op c neuropathies & Retrochiasma c visual pathway and cor cal lesions \\
\hline $\begin{array}{l}\text { - } \text { re nopathies secondary to vitamin } \\
\text { - degeneriency } \\
\text { the cone cells (cones) (tested by } \\
\text { electrore nogram ERG) } \\
\text { - toxic re nopathies } \\
\text { - re nopathies associated to } \\
\text { carcinoma } \\
\text { - re nopathies associated to } \\
\text { malignant melanoma }\end{array}$ & 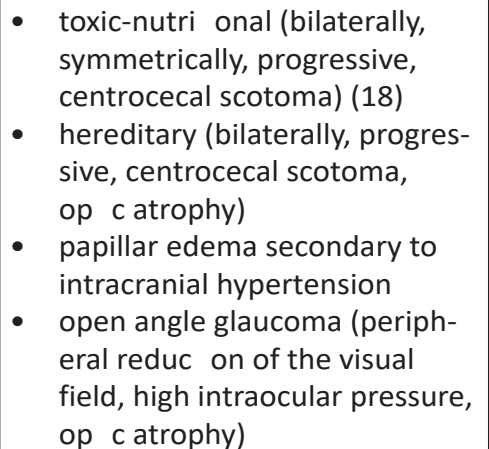 & $\begin{array}{l}\text { - } \quad \text { neurological signs and symptoms associated } \\
\text { bitemporal hemianopia due to compression } \\
\text { on the op c chiasma (hypophyseal adenoma, } \\
\text { meningioma, craniopharyngioma, aneurisms) } \\
\text { - cerebral MRI is necessary for the diagnosis } \\
\text { - homonymous hemianopia (affec ng the } \\
\text { contralateral op c tract pathway, corpus } \\
\text { geniculatum laterale, op c radia ons or the } \\
\text { visual cortex) } \\
\text { cor cal blindness (damage of occipital cortex } \\
\text { with normal eye - known as Anton Babinski } \\
\text { syndrome) }\end{array}$ \\
\hline
\end{tabular}




\section{CONCLUSIONS}

Visual acuity loss has different causes and pathophysiological mechanisms, with a great burden in everyday's life, severely affecting patients and their quality of life. Early diagnosis and specif-

\section{REFERENCES}

1. Allan Ropper, Martin Samuels, Joshua Klein. Adams and Victor's Principles of Neurology 10th Edition. McGraw Hill Professional, May 25, 2014

2. Newman N., Biousse V. Diagnostic approach to vision loss. Continuum (Minneap Minn). 2014 Aug; 20(4 Neuro-ophthalmology):785-815

3. Amick A., Caplan L.R. Transient monocular visual loss. Compr Ophthalmol Update. 2007 Mar-Apr. 8(2):91-8; discussion 99-100

4. Biousse V., Newman N. Retinal and optic nerve ischemia. Continuum (Minneap Minn). 2014 Aug; 20(4 Neuro-ophthalmology):838-56

5. Gundogan F.C., Yolcu U., Akay F. et al. Diabetic Macular Edema. Pak J Med Sci. 2016; 32(2):505-10

6. Al-Halafi A.M. Tremendous result of bevacizumab in malignant hypertensive retinopathy. Oman J Ophthalmol. 2015; 8(1):61-3

7. Hickman S.J., Dalton C.M., Miller D.H. et al. Management of acute optic neuritis. Lancet. 2002 Dec 14; 360(9349):1953-62.

8. Wingerchuk D.M., Lennon V.A., Lucchinetti C.F. et al. The spectrum of neuromyelitis optica. Lancet Neurol. 2007 Sep; 6(9):805-15.

9. Dutton J.J., Burde R.M., Klingele T.G. Autoimmune retrobulbar optic neuritis. Am J Ophthalmol. 1982 Jul; 94(1):11-7.

10. Ray S., Gragoudas E. Neuroretinitis. Int Ophthalmol Clin. 2001; 41(1):83-102 ic treatments are without any doubt the only way to reduce this discomfort. Ophthalmologists and neurologists should work together to overcome many problems of differential diagnosis.

Conflict of interest: none declared Financial support: none declared

11. Sundaram S.V., Purvin V.A., Kawasaki A. Recurrent idiopathic neuroretinitis: natural history and effect of treatment. Clin Exp Ophthalmol. 2010 Aug; 38(6):591-6

12. Gray A.V., Reed J.B., Wendel R.T. et al. Bartonella henselae infection associated with peripapillary angioma, branch retinal artery occlusion, and severe vision loss. Am J Ophthalmol. 1999 Feb; 127(2):223-4.

13. Costello F. Inflammatory optic neuropathies. Continuum (Minneap Minn). 2014 Aug; 20(4 Neuro-ophthalmology):816-37

14. Kouge J., Hayashida S., Imaki H. et al. A Japanese case of neuroborreliosis with papillitis. Rinsho Shinkeigaku. 2015; 55(4):248-53

15. Träisk F., Lindquist L. Optic nerve involvement in Lyme disease. Curr Opin Ophthalmol. 2012 Nov; 23(6):485-90

16. Takahashi T., Tamura M., Takasu T. The PCR-Based Diagnosis of Central Nervous System Tuberculosis: Up to Date. Tuberc Res Treat. 2012; 2012:831292

17. Yang H.K., Park M.J., Lee J.H. et al. Incidence of toxic optic neuropathy with low-dose ethambutol. Int J Tuberc Lung Dis. 2016 Feb; 20(2):261-4.

18. Mihailescu G., Buraga I., Nica S.M. et al. Manifestari neurologice in boli digestive. Ed Viata Medicala Romaneasca 2016. ISBN 978-973-160-094-9. pag 289, 332, 335-336 